\title{
Directly patterning metal films by nanoimprint lithography with low-temperature and low-pressure
}

\author{
H.L. Chen ${ }^{\mathrm{a}, *}$, S.Y. Chuang ${ }^{\text {a }}$, H.C. Cheng ${ }^{\mathrm{b}}$, C.H. Lin ${ }^{\mathrm{b}}$, T.C. Chu ${ }^{\mathrm{c}}$ \\ a Department of Materials Science and Engineering, National Taiwan University, Taiwan, ROC \\ ${ }^{\mathrm{b}}$ National Nano Device Laboratory, Hsinchu, Taiwan, ROC \\ ${ }^{\mathrm{c}}$ Yuanpei Institute of Science and Technology, Hsinchu, Taiwan, ROC
}

Available online 17 February 2006

\begin{abstract}
We demonstrated a new imprint method named nanoimprinting in metal/polymer bi-layer structures (NIMB) for patterning metal films with varied profiles. Converse with conventional nanoimprint lithography, the patterned mold is directly imprint in metal films not in polymer based resists. In general, direct imprint in metal films need ultra-high pressure or temperature to form patterns. In this paper, we improve the direct imprint processes by using a sharp mold and an underlying soft pad layer for the reduction of the imprint pressure and temperature. The imprint pressure can be reduced to be compatible with the conventional nanoimprint instrument. For the direct contact the metal film with mold, no surfactant should be coated on the surface of mold. It also indicates no mold-rework-processes are necessary for this direct imprint method.
\end{abstract}

(C) 2006 Elsevier B.V. All rights reserved.

Keywords: Nanoimprint lithography; Mold; Metal/polymer bi-layer structure; Optical grating

\section{Introduction}

Nanoimprint lithography (NIL), a potential candidate for the next generation lithography technology, has become more and more popular because of its low-cost, high-throughput and high-resolution [1]. As shown in Fig. 1(a), NIL defines patterns by physically deformation of deformable polymer materials (resist) by adding temperature above their glass transition temperature $\left(T_{\mathrm{g}}\right)$. During the imprint process, the pattern of the mold is duplicated into the resist by an air-press or oil-press type instrument. The conventional method for patterning the imprint molds is electron-beam lithography and dry etching processes.

Pattering varied profiles of metal films with sub-micrometer scale is important for the fabrication of optoelectronic and bio-photonic devices, such as optical gratings, reflective polarizer, and grating couplers. Many kinds of metal

\footnotetext{
${ }^{*}$ Corresponding author.

E-mail address: hsuenlichen@ntu.edu.tw (H.L. Chen).
}

films such as gold, silver, and copper are difficult to pattern by dry-etching processes. Generally, the metal patterns are defined by lift-off or wet-etching processes that are more complicated and are not reproducible. Recently, a process for direct imprint of gold films was reported and shown in Fig. 1(b). The experiment was carried out at room temperature with ultra-high pressure (several hundreds $\mathrm{MPa}$ ) by using an oil-press imprint instrument [2]. This technology is highly appreciated to fabricate optical elements such as reflective polarizer on gold films or optoelectronic devices [3]. But such processes with ultra-high pressure are not desirable that would damage the underlying substrates or devices. The air-press type imprint instrument can provide uniform pressure with large substrate-size [4]. However, it can not be direct imprint in metal films because of the relative low-pressure limitation (about $10 \mathrm{MPa}$ ).

In this paper, we demonstrated a new imprint method named nanoimprinting in metal/polymer bi-layer structures (NIMB) for patterning metal films with varied profiles. As shown in Fig. 1(c), we improve the direct imprint 


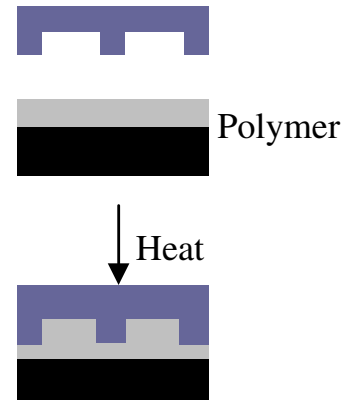

(a)
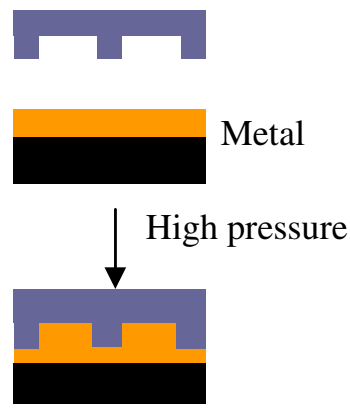

(b)
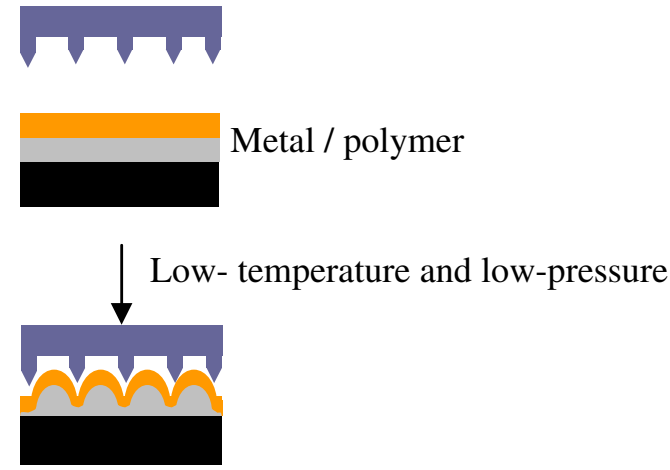

(c)

Fig. 1. Schematic diagrams of imprint technologies (a) conventional nanoimprint lithography, (b) direct imprint metal films, (c) nanoimprint in metal/polymer bi-layer (NIMB).

processes by using a sharp mold and an underlying soft pad layer for the reduction of the imprint pressure and temperature. By suitable baking processes, the imprint pressure can be reduced to be compatible with the conventional air-press or oil-press type imprint instrument. The imprint pressure of our NIMB method is only about $1 \%$ comparing with the previous directly imprinting metal method [2]. One of the issues in conventional nanoimprint lithography is that the resist would be stick on the mold during the imprinting process. Therefore, suitable surfactant should be coated on the surface of mold to isolate the sticky resist. In this paper, the mold directly contacts with the metal film; no surfactant should be coated on the surface of mold. It also indicates that there is no mold-rework-process requirement for this NIMB method.

\section{Experiment}

The diagram of metal film stack and the imprint mold were shown in Fig. 1(c). We applied the commercial resist NEB-22 (Sumitomo, $T_{\mathrm{g}}=105^{\circ} \mathrm{C}$ ) or SU-8 (Microchem, $T_{\mathrm{g}}=55^{\circ} \mathrm{C}$ ) as the underlying pad layer on the silicon substrate. Then the thermal evaporator system was used to deposit gold and Pt-Pd films with thickness $30-50 \mathrm{~nm}$ on the resist coated substrate. The imprint process was carried out by an air-press type instrument (Nanonex, NX-1000) or a conventional oil-press type imprint machine. The imprint pressure and heating temperature of imprint processes were about $4-20 \mathrm{MPa}$ and $40-80{ }^{\circ} \mathrm{C}$, respectively. The silicon mold that be used in our experiments was fabricated by using the electron-beam lithography (Leica, Weprint-200) followed by the reactive-ion-etching process. The high-density-plasma reactive-ion-etching (HDP-RIE) system (Duratek, Mutiplex Cluster) with inductively coupled plasma (ICP) sourced was used to fabricate molds with varied profiles. The images of patterned metal films were measured by optical microscope and scanning electron microscope (Hitachi, S-4000).

\section{Results and discussion}

\subsection{The application of sharp molds}

The direct imprint metal films method is undesirable for the ultra-high imprinting pressure. To overcome this problem, the sharp mold was used to increase the tippressure then the requirement of imprint pressure can be reduced. The silicon mold used in our experiments was fabricated by using electron-beam lithography followed by the HDP-RIE process. Fig. 2(a) shows the conventional binary silicon mold with flattop. The bias and RF power of etching processes are the critical parameters for mold profile controlling. As shown in Fig. 2(b), the sharp mold can be fabricated by optimization the etching processes. The HDP-RIE system with ICP-source has a chamber surrounded by RF coils and a RF-bias provided for substrates. The former enables a high-density plasma with a large ion content and the latter can control the energy of ion-bombardment through a DC bias arises. For the fabrication of sharp mold, we use the $400 \mathrm{~W}$ RF-bias power that would increase ion-bombardment
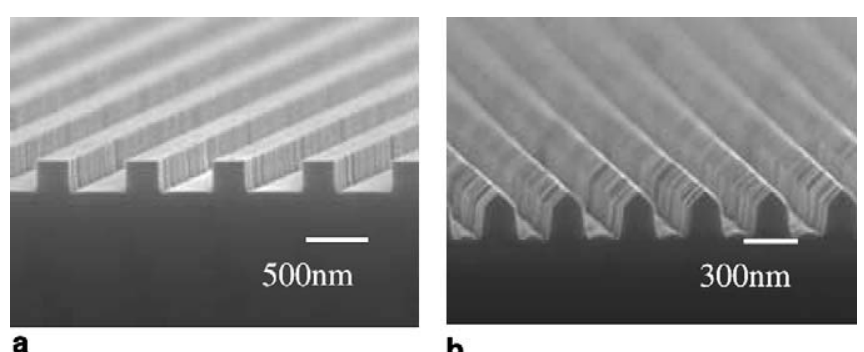

b

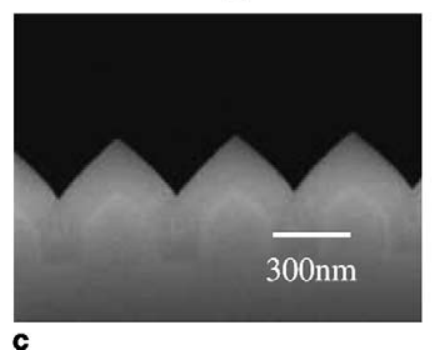

Fig. 2. SEM images of molds (a) the conventional binary mold with flattop, (b) the sharp mold, (c) the triangle mold. 

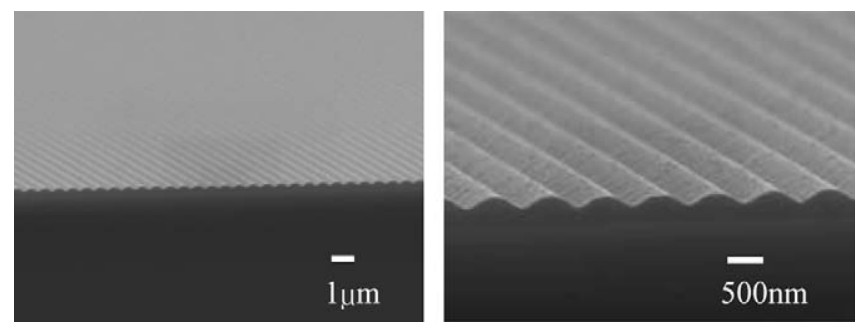

Fig. 3. SEM images of the cross-section of the gold film stack patterned by using the NIMB method.

on the surface of the mold. As shown in Fig. 2(c), we can also fabricate the triangle mold by the autocloning effect of HDP-chemical vapor deposition (CVD) system [5,6]. Fig. 3 shows the SEM images of the cross-section of gold films stack patterned by using direct imprint method with sharp molds.

\subsection{The influence of imprint temperature}

For the general NIL process, the heating temperature is about $20-30{ }^{\circ} \mathrm{C}$ above the glass transition temperature $\left(T_{\mathrm{g}}\right)$ of resists to ensure the resist become flow. In our NIMB processes, if the imprint temperature $\left(130^{\circ} \mathrm{C}\right)$ over $T_{\mathrm{g}}$ $\left(105^{\circ} \mathrm{C}, \mathrm{NEB}-22\right)$ of the underlying resist layer, the flowing resist would overflow to the surface of gold films and caused poor pattern quality as shown in Fig. 4(a). Because the thickness of gold films is only $40 \mathrm{~nm}$, flowing resist are effused from gold thin films during compressing process. This result indicates that the temperature of the directly imprint process must be maintained below the glass transition temperature of the underlying soft pad to avoid pattern distortion. Fig. 4(b) shows the good image profile with $60{ }^{\circ} \mathrm{C}$ during direct imprint metal films process. Similarly, we found the optimal imprint temperature is about $45^{\circ} \mathrm{C}$ for the SU-8 resist $\left(T_{\mathrm{g}}=55^{\circ} \mathrm{C}\right)$ as the underlying layer.

\subsection{The influence of the imprinting pressure}

Fig. 3 shows the SEM image of the cross-section of gold films stack patterned by using the NIMB method. The imprint pressure is only about $1 \%$ comparing with the pre-
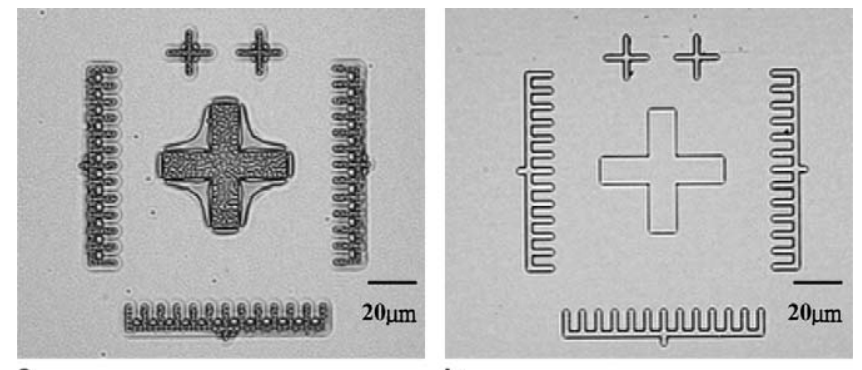

a

b

Fig. 4. Optical microscopic images of gold film stacks with different imprint temperature (a) $130{ }^{\circ} \mathrm{C}$, (b) $60^{\circ} \mathrm{C}$.

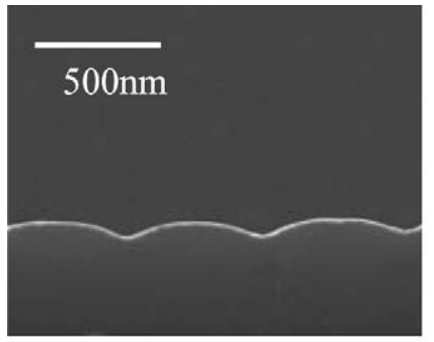

a

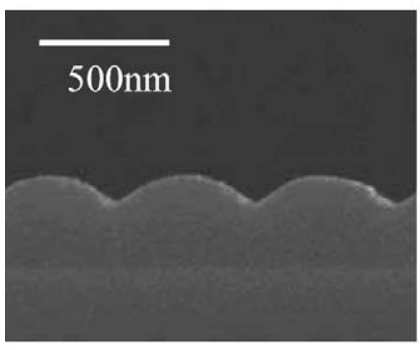

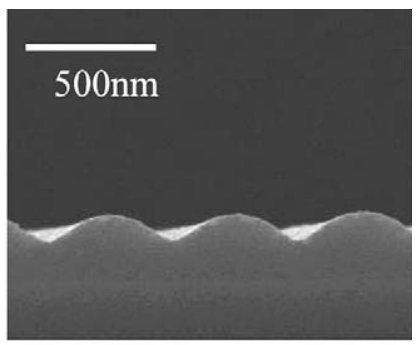

b

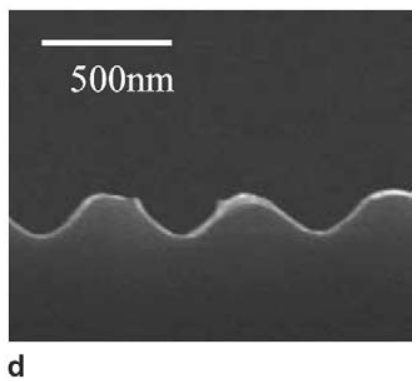

Fig. 5. The depth of surface-profile in metal films can be tuned by using different imprint pressure (a) $7 \mathrm{MPa}$, (b) $12 \mathrm{MPa}$, (c) $14 \mathrm{MPa}$, (d) $17 \mathrm{MPa}$.
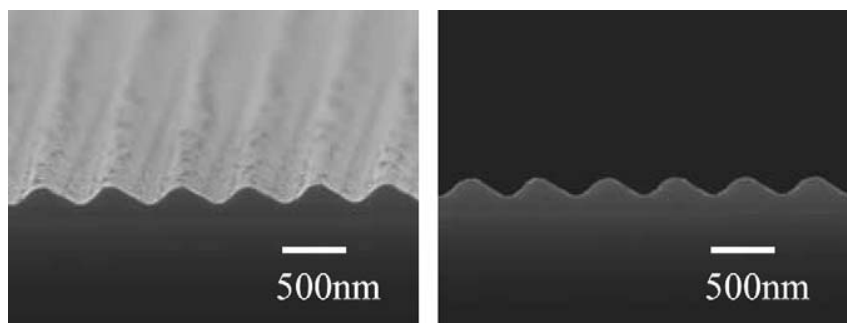

Fig. 6. Cross SEM images of blazed gratings can be fabricated by the NIMB method.

vious method. The surface of the gold films stack was found with sinusoidal variation. According to this result, we can obtain the desired curvature of shape by using different imprint pressure and different shape of molds. Fig. 5 shows the depth of surface-profile in gold films can be tuned by using different imprint pressure. As shown in Fig. 6, we also found that the blazed gratings can also be fabricated by using this directed imprint method with the blazed mold and suitable imprint pressure.

\subsection{The influence of the properties of metal films}

To demonstrate the effect of different metal films, the gold films and Pt-Pd alloy films with the same $30 \mathrm{~nm}$ thickness were prepared for the NIMB method. The gold films are higher ductility and lower hardness than Pt-Pd alloy films. Therefore, the depth of surface-profile in the gold film is deeper than the Pt-Pd film as show in Fig. 7. Although the gold film has better imprint depth, the gold film was also found easily broken with the same imprintpressure for the gold film with lower ultimate tensile strength than the Pt-Pd alloy film [7]. Therefore, the prop- 


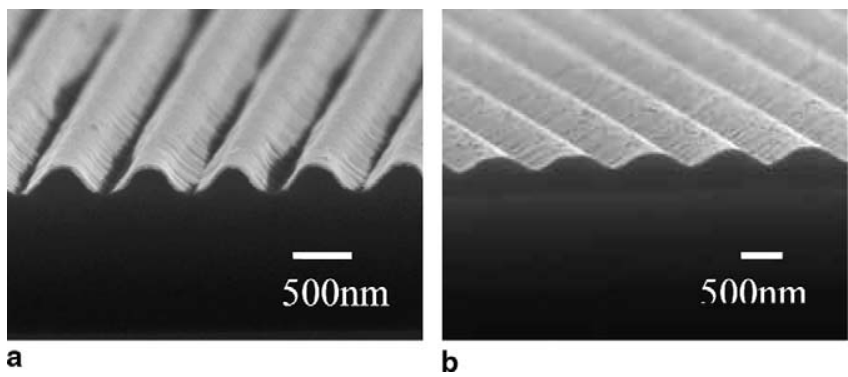

Fig. 7. SEM images of (a) gold films (b) Pt-Pd alloy films with the same thickness and pressure in the NIMB method.

erties of metal films are also the important factors in the NIMB method.

\section{Conclusion}

We demonstrate a new imprint method named nanoimprinting in metal/polymer bi-layer structures (NIMB) for patterning metal films with varied profiles. In this paper, we improve the direct imprint processes by using a sharp mold and an underlying soft pad layer for the reduction of the imprint pressure and temperature. The imprint pressure can be reduced to be compatible with the conventional air-press or oil-press type nanoimprint instrument. The HDP-RIE system can be used to fabricate molds with varied profiles. We can obtain the desired curvature of shape by using different imprint pressure and different shape of molds. For direct contact the metal films with mold, no surfactant should be coated on the surface of mold. It also indicates no mold-rework-processes requirement for this direct imprint method. We also find the properties of metal films are the important factors in the NIMB method.

\section{Acknowledgments}

The authors are very thankful to the National Science Council, Taiwan, ROC for supporting this study under project NSC94-2215-E-002-026.

\section{References}

[1] S.Y. Chou, P.R. Krauss, P.J. Renstrom, Science 272 (1996) 85.

[2] H. Yoshihiko, U. Toshihiko, K. Tomohiro, M. Takashi, SPIE - Int. Soc. Opt. Eng. 74 (2003) 5220.

[3] B.E.A. Saleh, M.C. Teich, Fundamentals of Photonics, Wiley, New York, 1991.

[4] Mingtao Li, Hua Tan, Linshu Kong, Larry Koecher, SPIE - Int. Soc. Opt. Eng. (2004).

[5] M. Notomi, T. Tamamura, T. Kawashima, S. Kawakami, Appl. Phys. Lett. 77 (2000) 4256-4258.

[6] H.L. Chen, H.F. Lee, W.C. Chao, C.I. Hsieh, F.H. Ko, T.C. Chu, J. Vac. Sci. Technol. B 22 (6) (2004) 3359-3362.

[7] H.D. Espinosa, B.C. Prorok, J. Mater. Sci. 38 (2003) 4125-4128. 